\title{
Merkezi İşlem Biriminde Elektromanyetik Dalga Yayılımı Davranışı
}

\author{
Ayşe Nihan Basmac1 ${ }^{1 *}$, Seçkin Filiz ${ }^{2}$

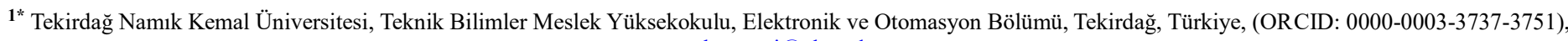 \\ anbasmaci@nku.edu.tr \\ 2 Tekirdağ Namık Kemal Üniversitesi, Teknik Bilimler Meslek Yüksekokulu, Makine ve Metal Teknolojileri Bölümü, Tekirdağ, Türkiye (ORCID: 0000-0002-9383- \\ 8915), sfiliz@nku.edu.tr
}

(2nd International Conference on Access to Recent Advances in Engineering and Digitalization (ARACONF)-10-12 March 2021)

(DOI: 10.31590 /ejosat.898242)

ATIF/REFERENCE: Basmacı, A. N. \& Filiz, S. (2021). Merkezi İşlem Biriminde Elektromanyetik Dalga Yayılımı Davranışı. Avrupa Bilim ve Teknoloji Dergisi, (24), 5-9.

$\ddot{O} \mathbf{z}$

Bu çalışmada, izotropik katmanlara sahip merkezi işlem birimlerinde elektromanyetik dalga yayılımı davranışı incelenmektedir. Ayrıca, merkezi işlem birimlerinin katmanlı yapılarının ve katmanların malzeme özelliği parametrelerinin enine elektrik (TE) ve enine manyetik (TM) modlar için elde edilen alan dağı̆lımları üzerindeki etkileri araştırılmaktadır.

Anahtar Kelimeler: Enine elektrik (TE) mod, Enine manyetik (TM) mod, Elektromanyetik dalga yayılımı, Merkezi işlem birimi (CPU).

\section{The Behaviour of Electromagnetic Wave Propagation in a Central Processing Unit}

\begin{abstract}
In this study, the behavior of electromagnetic wave propagation in central processing units with isotropic layers is investigated. In addition, the effects of the central processing units' layered structures and the material property parameters of the layers on the field distributions obtained for the transverse electric (TE) and transverse magnetic (TM) modes are examined.
\end{abstract}

Keywords: Transverse electric (TE) mode, Transverse magnetic (TM) mode, Electromagnetic wave propagation, Central processing unit (CPU).

\footnotetext{
* Ayşe Nihan Basmacı: Tekirdağ Namık Kemal Üniversitesi, Teknik Bilimler Meslek Yüksekokulu, Elektronik ve Otomasyon Bölümü, Tekirdağ, Türkiye, ORCID: 0000-0003-3737-3751, anbasmaci@nku.edu.tr
} 


\section{Giriş}

Maxwell denklemlerinin çözümünde de kullanılan zaman uzayda sonlu farklar yöntemi (FDTD) ya da bir diğer ismiyle Yee Yöntemi 1966 y1lında geliştirilmiştir (Yee, 1966). Bu yöntem özellikle bir, iki ve üç boyutlu yapılarda meydana gelen elektromanyetik dalga yayılımı davranışının belirlenmesi için kullanılmaktadır (Sullivan, 2000). Optik, optoelektronik ve fiber optik teknolojilerinde yaşanan önemli gelişmelerle birlikte, fotonik yapıların ve dalga kılavuzlarının kullanımı da yaygın hale gelmiştir. Fotonik yapılar farklı optik özelliklere sahip malzemelerin periyodik olarak, yanyana konumlandırılmasıyla oluşturulabilirler. (Johnson vd., 2013; El Haddad, 2016). Aynı ya da farklı malzeme özelliklerine sahip dalga kılavuzlarının art arda konumlandırılmasıyla, elektromanyetik dalga yayılımı davranışı ayarlanabilmektedir (Soltani vd., 2017; Hirani vd., 2018; Basmaci, 2020; Panyaev vd., 2020). Elektromanyetik dalga yayılımı davranışının ayarlanabilmesi için kullanılan tüm fotonik yapı katmanları birbirleriyle aynı malzeme özelliklerine sahip olabileceği gibi, birbirlerinden tamamen farklı malzeme özelliklere de sahip olabilirler (Cho vd., 2020; Zhang vd., 2021).

Taşınabilir bilgisayarlar ve cep telefonlarının taşınabilirlik anlamında katettiği ilerlemede merkezi işlem biriminin (CPU) tasarımı konusunun da önemli bir rolü vardır. CPU tasarlanırken, tasarlanan yapıda hızlı ısınmanın engellenebilmesi için açığa çıkan 1sı enerjisinin uygun bir biçimde dağıtılması gerekliliği, yapının tasarımı esnasında göz önünde bulundurulması gereken konular arasında başı çekmektedir. Malzeme özelliği parametrelerinin elektrik alan dağılımı üzerindeki etkilerinin incelendiği bir çalışmanın da bu konuya katkı sağlayabileceği düşünülmektedir (Zhu vd., 2011). Ayrıca, CPU'da ve grafik işlem biriminde (GPU) meydana gelen elektromanyetik dalga yayılımı davranışının FDTD yöntemi kullanılarak incelendiği (Xiong vd., 2018) bir çalışma ve FDTD yöntemiyle elektromanyetik radyasyonun insan üzerindeki etkilerinin incelendiği (Kaburcuk \& Elsherbeni, 2018; Lwin \& Yokota, 2019) bir diğer çalışma da CPU tasarımına katkı sağlayabilecek çalışmalar arasındadır.

$\mathrm{Bu}$ çalışmada, TE ve TM modları için farklı malzeme özelliklerine sahip 6 adet katmanın bir araya getirilmesiyle oluşturulmuş CPU'larda meydana gelen elektromanyetik dalga yayılımı davranışı incelenmektedir. Bu amaçla, CPU'ların TE ve TM modları için alan dağılımları belirlenmektedir. İncelenen CPU'lar, literatürdeki diğer çalışmalarda incelenen CPU'lardan (Bouras vd., 2018; Duman \& Kaburcuk, 2019; Mitri, 2020; Panyaev vd., 2020) yapisal anlamda farklıdir.

\section{Materyal ve Metot}

Merkezi işlem birimine (CPU) ait lineer, homojen, izotrop katmanlardan biri Şekil 1'de görülmektedir.

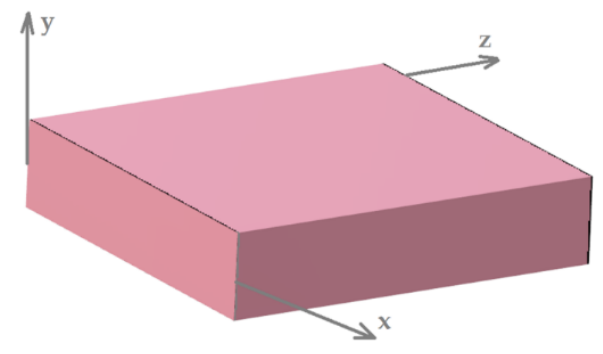

Şekil 1. CPU'ya ait, lineer, homojen, izotrop katman
Lineer, homojen ve izotrop ortamda, Maxwell denklemleri aşağıdaki gibi ifade edilmektedir (Pozar, 2016):

$$
\begin{gathered}
\nabla \cdot \vec{E}=0 \\
\nabla \cdot \vec{H}=0 \\
\nabla \times \vec{E}=-i \omega \mu \vec{H} \\
\nabla \times \vec{H}=-i \omega \varepsilon \vec{E}
\end{gathered}
$$

burada, $\mu$ manyetik geçirgenlik sabiti, $\varepsilon$ dielektrik sabiti, $\vec{E}$ elektrik alan vektörü ve $\vec{H}$ manyetik alan vektörüdür. TE modu için elektromanyetik dalga yayılımı denklemi, Denk. (1c) ve Denk. (1d) kullanılarak aşağıdaki gibi elde edilmektedir:

$$
\nabla \times(\nabla \times \vec{H})=\nabla(\nabla \cdot \vec{H})-\nabla^{2} \vec{H}=\nabla \times\left(-\mu \frac{\partial \vec{H}}{\partial t}\right)
$$

Denk. (2)'nin çözümü sonucunda elde edilen zaman ve konuma bağlı kısmi diferansiyel denklem aşağıdaki gibidir:

$$
\frac{\partial^{2} H_{Z}}{\partial x^{2}}+\frac{\partial^{2} H_{Z}}{\partial y^{2}}-\mu \varepsilon \frac{\partial^{2} H_{z}}{\partial t^{2}}=0
$$

burada, $\mu \varepsilon$ elektromanyetik malzeme özelliği parametresini temsil etmektedir. TM modu için de elektromanyetik dalga yayılımı denkleminin benzer biçimde elde edilebilmesi mümkündür. Elektromanyetik dalga yayılımı davranışı incelenen merkezi işlem birimine ait katmanlardan biri Şekil 1'de, bu katmanda meydana gelen elektromanyetik dalga yayılımı Şekil 2 'de görülmektedir. Şekillerden de görüldüğü gibi, incelenen CPU katmanı dalga kılavuzu olarak kullanılmaktadır.

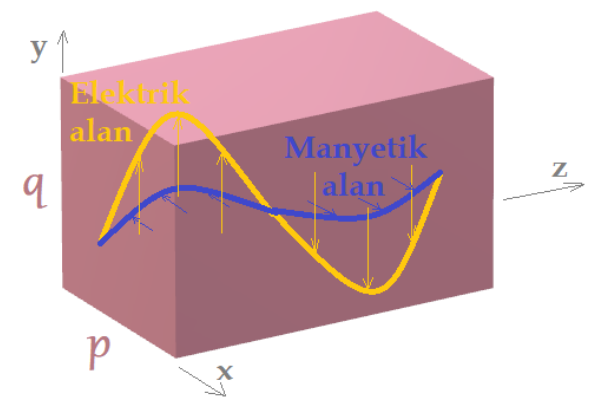

Şekil 2. Merkezi işlem birimine ait, lineer, homojen, izotrop katmanda meydana gelen elektromanyetik dalga yayılımı

$\mathrm{TM}_{\mathrm{mn}}$ modunda, $\mathrm{H}_{\mathrm{Z}}=0$ olduğu durumda, $\mathrm{E}_{\mathrm{Z}}$ aşağıdaki gibi ifade edilmektedir:

$$
\mathrm{E}_{z}=D_{T M}\left(\sin \frac{m \pi x}{p}\right)\left(\sin \frac{n \pi y}{q}\right) e^{i \omega t}
$$

burada, $D_{T M}$ TM mod için genlik sabitini, $m$ ve $n$ sırasıyla, x ve y eksenleri için dalga sayılarını temsil etmektedir.

$\mathrm{TE}_{\mathrm{mn}}$ modunda, $\mathrm{E}_{\mathrm{Z}}=0$ iken, $\mathrm{H}_{\mathrm{Z}}$ aşağıdaki şekilde ifade edilmektedir:

$$
\mathrm{H}_{z}=D_{T E}\left(\cos \frac{m \pi x}{p}\right)\left(\cos \frac{n \pi y}{q}\right) e^{i \omega t}
$$

burada, $D_{T E}$ TE mod için genlik sabitini temsil etmektedir.

Şekil 2'de de görülen CPU'ya ait lineer, homojen ve izotrop katmanının TM ve TE modları için, Denk. (4) ve Denk. (5)'in kesin çözümüyle elde edilen alan dağılımları Şekil 3 'teki gibidir. 

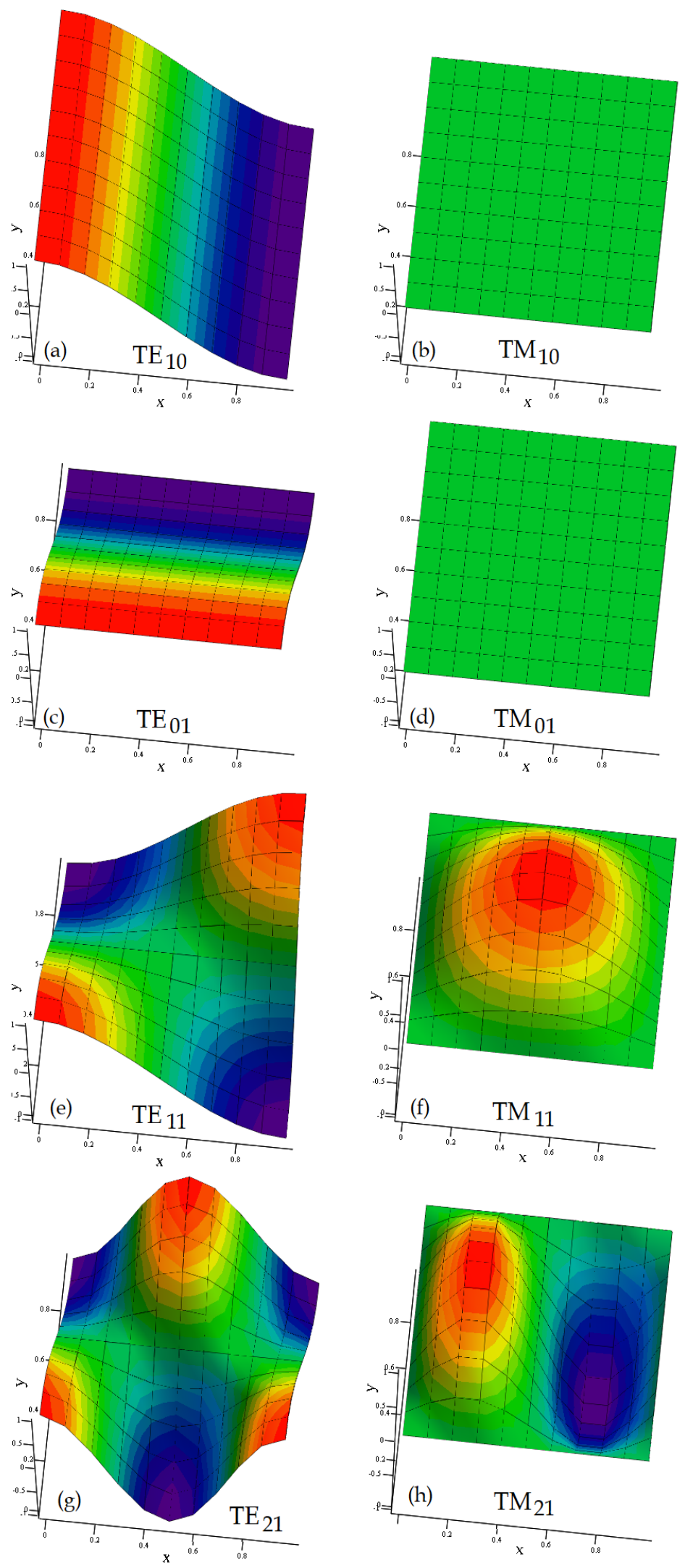

Şekil 3. Lineer, homojen ve izotrop CPU katmanında TE ve TM modları için alan dağılımları, (a) $T E_{10}$, (b) $T M_{10}$, (c) $T E_{01}$, (d) $T M_{01}$, (e) $T E_{11}$, (f) $T M_{11}$, (g) $T E_{21}$ ve (h) $T M_{21}$

Denk. (4) ve Denk. (5)'in kesin çözümüyle TE ve TM modları için elde edilen bu alan dağılımları FDTD yöntemiyle Şekil 4'te verilen düğümler kullanılarak da elde edilebilir.

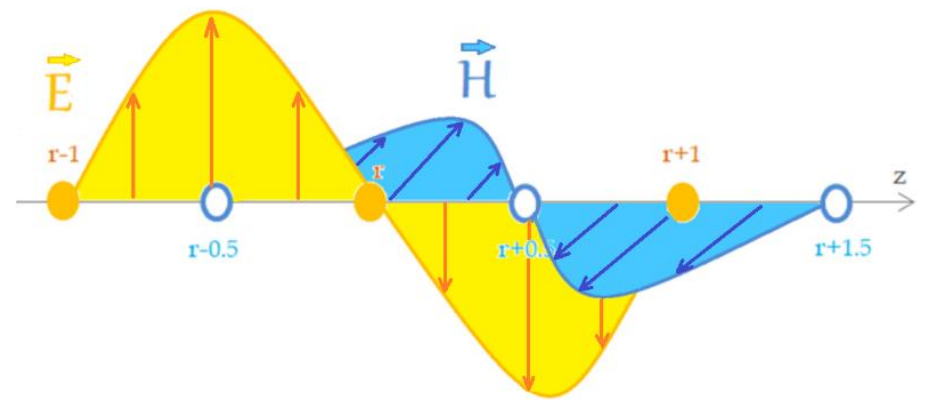

Şekil 4. FDTD yönteminin düğ̈̈mlerle (r) gösterimi

FDTD yöntemiyle Şekil 4'te verilen düğümler kullanılarak Denk. (3)'ün çözümü, Tablo 1'de verilen ifadeler kullanılarak yapılabilir (Sullivan, 2000 ve Yee, 1966):

Tablo 1. FDTD çözümü için gerekli türev ifadeleri

\begin{tabular}{c|c}
\hline Türev İfadesi & Sonlu Farklar ifadesi \\
\hline$H$ & $\frac{h_{r}}{\partial H} \frac{h_{r+s}-h_{r-s}}{2 \Delta r}$ \\
\hline$\frac{\partial r}{\partial^{2} H} \frac{\partial r^{2}}{}$ & $\frac{h_{r+s}-2 h_{r}+h_{r-s}}{\Delta r^{2}}$ \\
\hline
\end{tabular}

Tablo 1'de $r$ değeri $x, y$ veya t boyutlarından birinde birer adım aralığı ile artışı, $\Delta r$ birbirine komşu olan iki düğüm arasındaki mesafeyi, $s$ değeri ise $x, y$ veya t boyutlarından birinde 0.5 'er adım aralığı ile artışı temsil etmektedir.

\section{Bulgular ve Tartışma}

Bu çalışmada, farklı biçimde tasarlanmış iki adet CPU'nun $\mathrm{TE}_{11}$ ve $\mathrm{TM}_{11}$ modları için alan dağılımları elde edilmektedir. Alan dağılımı incelenen ilk CPU'da (CPU I), CPU katmanları Tablo 2 'de verilen sıralamayla Şekil 5 'teki gibi konumlandırılmakta ve malzeme özelliği parametreleri de yine tabloya göre belirlenmektedir. Alan dağılımı incelenen ikinci CPU'da (CPU II), CPU katmanlarının sıralaması ve malzeme özelliği parametreleri, birinci CPU'dan $\mathrm{TM}_{11}$ modu için elde edilen alan dağılımının tam zıttı bir alan dağılımı elde edilmek üzere belirlenmektedir.

Tablo 2. CPU I'ait katman siralamasl ve her bir katmanda kullanilan malzemeler

\begin{tabular}{c|c|c}
\hline No & Katmanlar & Malzeme \\
\hline 1 & Taban & FR4 epoksi \\
\hline 2 & Entegre devre & $\mathrm{SiO}_{2}$ \\
\hline 3 & Entegre 1Sı dağıtıcı & $\mathrm{Al}$ \\
\hline 4 & Termal macun & $\mathrm{Silikon}$ \\
\hline 5 & Entegre devre bağlantı malzemesi & $\mathrm{Ag}$ \\
\hline 6 & IHS dolgu macunu & Epoksi \\
\hline
\end{tabular}

Bu iki CPU'nun, Şekil 6'da ve Şekil 7'de de görülen, TE ve TM modlarına ilişkin alan dağılımları kıyaslandığında, CPU'ların katmanlarındaki sıralamayla, bu katmanların malzeme özelliği parametrelerindeki değişimin alan dağılımları üzerindeki etkisinin TE modlarına kıyasla TM modlarında oldukça fazla sonucuna ulaşılmaktadır. Alan dağılımları, FDTD 
yöntemini kullanan ANSYS Lumerical programı ile elde edilmektedir.

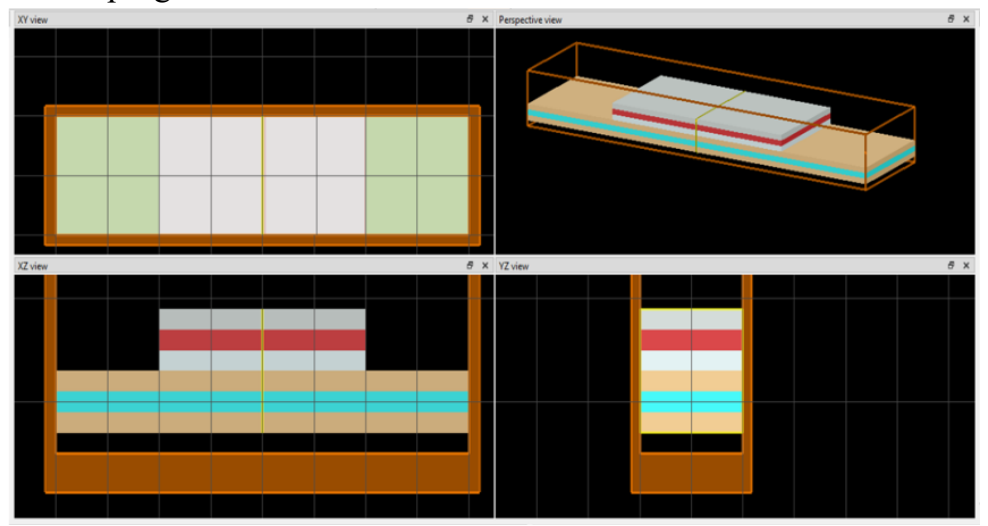

Şekil 5. CPU I'in katmanlarının dizilimi
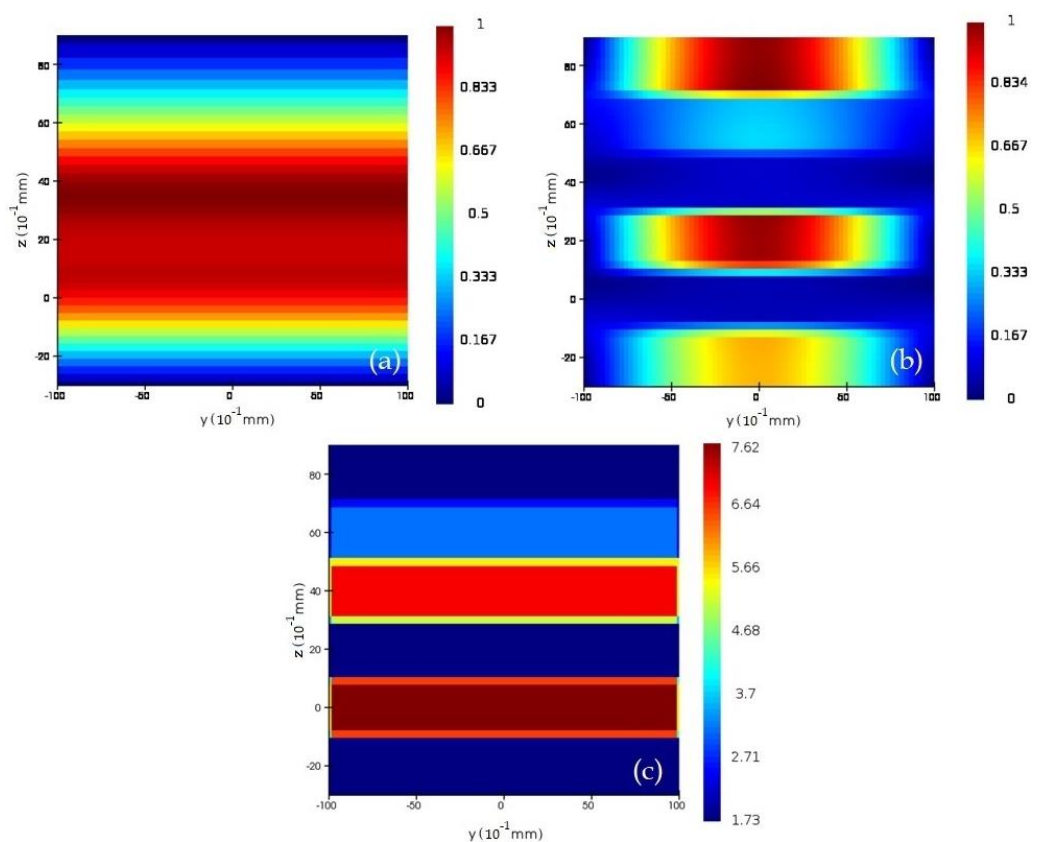

Şekil 6. CPU I'in, (a) TE modu için alan dağllımı, (b) TM modu için alan dă̆ılımı, (c) malzeme özelliği parametreleri
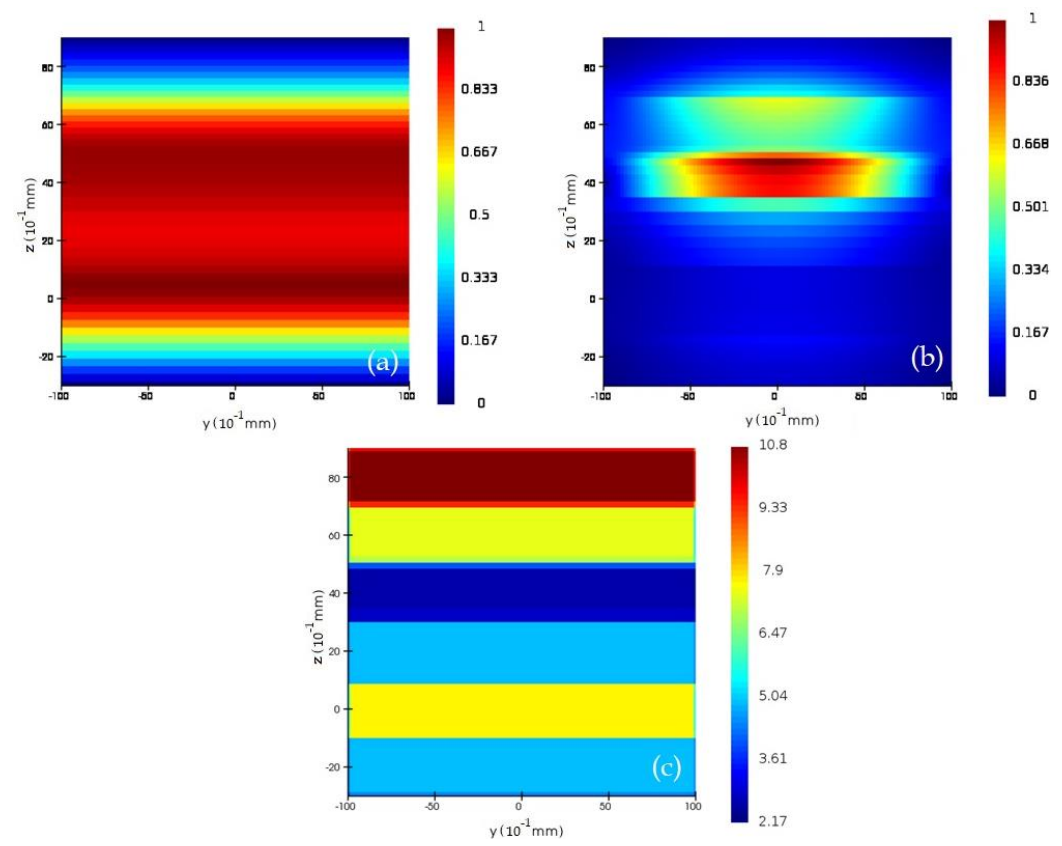

Şekil 7. CPU II'nin, (a) TE modu için alan dağıllımı, (b) TM modu için alan dăğlımı, (c) malzeme özelliği parametreleri 


\section{Sonuç}

$\mathrm{Bu}$ çalışmada, izotropik katmanlara sahip merkezi işlem birimlerinde meydana gelen elektromanyetik dalga yayılımı davranışı incelenmektedir. Lineer, homojen ve izotrop katmanlara sahip iki farklı CPU'nun TE ve TM modları için alan dağılımları incelenmektedir. İncelenen CPU'ların katmanlarının dizilimleri ve malzeme özelliği parametreleri birbirinden farklıdır. CPU'ların katmanlarındaki dizilimin ve bu katmanların sahip olduğu malzeme özelliği parametrelerinin, yapıların TE ve TM modları için elde edilen alan dağılımları üzerindeki etkileri araştırılmaktadır. FDTD yöntemini kullanan ANSYS Lumerical programı ile elde edilen alan dağılımları incelendiğinde, TM modları için elde edilen alan dağılımlarının TE modlarına kıyasla, CPU katmanlarının dizilimindeki ve malzeme özelliği parametrelerindeki değişimden daha fazla etkilendiği sonucuna ulaşılmaktadır.

İleride yapılacak olan çalışmalarda, her bir katmanı anizotrop özelliğe sahip olan, katmanlı yapıların, alan dağılımlarının aynı yöntemle incelenebilmesi mümkündür. Katmanlı yapıların katmanları arasındaki süreksizliklerin yani hasarların, yapıların alan dağılımları üzerindeki etkisi de araştırılması gereken konular arasındadır. Aşırı yüke ve yüksek sıcaklığa maruz bırakılan yapıların alan dağılımlarının belirlenmesi konusunda yapılacak olan çalışmalar da bu anlamda literatürdeki eksiği gidermek adına önem teşkil etmektedir.

\section{Kaynakça}

Basmaci, A.N. (2020). Characteristics of electromagnetic wave propagation in a segmented photonic waveguide. Journal of Optoelectronics and Advanced Materials, 22, 452-460.

Bouras, M., Mezhoud, M., \& Hocini, A. (2018). Efficient magneto-optical TE/TM mode converter in a hybrid structure made with a $\mathrm{SiO}_{2} / \mathrm{ZrO}_{2}$ coated on an ion-exchanged glass waveguide. Optik, 157, 658-666.

https://doi.org/10.1016/j.ijleo.2017.11.169

Cho, J., Park, M.-S., \& Jung, K.-Y. (2020). Perfectly matched layer for accurate FDTD for anisotropic magnetized plasma. Journal of Electromagnetic Engineering and Science, 20(4), 277-284.

https://doi.org/10.26866/jees.2020.20.4.277

Duman, Ç., \& Kaburcuk, F. (2019). A numerical study of ZnO random lasers using FDTD method. Optik, 181, 993-999.

http://dx.doi.org/10.1016/j.ijleo.2018.12.136

El Haddad, A. (2016). Exact analytical solution for the electromagnetic wave propagation in a photonic band gabs material with sinusoidal periodicity of dielectric permittivity. Optik, 127, 1627-1629.

\section{http://dx.doi.org/10.1016/j.ijleo.2015.11.049}

Hirani, R.R., Pathak, S.K., Shah, S.N., \& Sharma D.K. (2018). Dispersion characteristics of dielectric tube waveguide loaded with plasma for leaky wave antenna application. International Journal of Electronics and Communications (AË̈), 83, 123-130.

http://dx.doi.org/10.1016/j.aeue.2017.08.019
Johnson, S.G., Oskooi, A. \& Taflove, A. (2013). Advances in FDTD Computational Electrodynamics Photonics and Nanotechnology, Artech House, UK.

Kaburcuk, F., \& Elsherbeni, A.Z. (2018). Temperature rise and SAR distribution at wide range of frequencies in a human head due to an antenna radiation. The Applied Computational Electromagnetics Society, 33(4), 367-372.

Lwin, Z.M., \& Yokota, M. (2019). Numerical analysis of SAR and temperature distribution in two dimensional human head model based on FDTD parameters and the polarization of electromagnetic wave. International Journal of Electronics and Communications (AË̈), 104, 91-98.

https://doi.org/10.1016/j.aeue.2019.03.010

Mitri, F.G. (2020). Optimal TM TE mode conversion contribution to the radiation force on a cylinder exhibiting rotary polarization in circular polarized light. Journal of Quantitative Spectroscopy \& Radiative Transfer, 253, 107115 .

https://doi.org/10.1016/j.jqsrt.2020.107115

Panyaev, I., Zolotovskii, I., \& Dmitry, S. (2020). Laser generation and amplication of TE and TM modes in a semiconductor optical GaAs waveguide with distributed feedback generated by a space charge wave. Optics Communications, 459, 125026.

https://doi.org/10.1016/j.optcom.2019.125026

Pozar, D.M. (2012). Microwave Engineering $4^{\text {th }}$ Edition, John Wiley \& Sons, Inc. Amherst, Massachussetts.

Soltani, A., Ouerghi, F., Abdelmalek, F., Haxha, S., Ademgil, H., \& Akowuah, E.K. (2017). Unidirectional light propagation photonic crystal waveguide incorporating modified defects. Optik, 130, 1370-1376.

https://doi.org/10.1016/j.ijleo.2016.11.179

Sullivan, D.M. (2000). Electromagnetic Simulation Using The FDTD Method. IEEE Press, New York.

Xiong, L.-L, Wang, X.-M. Peng, Z.-Y. \& Zhong, S.-Y. (2018). The electromagnetic waves propagation in unmagnetized plasma media using parallelized finite-difference time-domain method. Optik, 166, 8-14.

https://doi.org/10.1016/j.ijleo.2018.03.136

Yee, K.S. (1966). Numerical solution of initial boundary value problems involving Maxwell's equations in isotropic media. IEEE Transactions on Antennas and Propagation, 11(3), 302307.

https://doi.org/10.1109/TAP.1966.1138693

Zhang, Y., Cao, L., Shi, D., \& Lin, Y. (2021). Multiscale analysis and algorithm of transient electromagnetic scattering from heterogeneous materials. Journal of Computational and Applied Mathematics, 391, 113427.

https://doi.org/10.1016/j.cam.2021.113427

Zhu, Y., Lu, J., \& Li, E. (2011). Electromagnetic compatibility benchmark-modeling approach for a dual-die CPU. IEE Transactions on Electromagnetic Compatibility, 53(1), 9198. https://doi.org/10.1109/TEMC.2010.2053208 